\title{
IE'12 awards by JAISE
}

During the 8th edition of the International Conference on Intelligent Environments (IE'12) held in Guanajuato (Mexico) last July, IOS Press provided rewarded several researchers by their attention to quality and the potential impact of their work. Prize to the best paper, demo and Poster were supported by JAISE and the AISE book series.

The prize for the best paper consists of one year free subscription to JAISE and other products accessible through MetaPress kindly provided by IOS Press, the publisher of JAISE. The chairs of the conference directly based upon the judgment of the Program Committee unanimously decided to award the prize to the paper: "An Approach for Secure Role Assignment" by Wolfgang Apolinarski, Marcus Handte, Pedro José Marrón. The photo in Fig. 1 shows, from left to right, Victor Zamudio Rodriguez (General Chair of IE'12), and Juan Carlos Augusto (Program co-Chair of IE'12) presenting a certificate to co-author Wolfgang Apolinarski.

The prize for the best Poster was awarded to: "Intelligent Event Management with Bluetooth Sensors Networks" by M. Versichele, R. Huybrechts, T. Neutens, and N. Van de Weghe. The photo in Fig. 2 shows, from left to right, Victor Zamudio Rodriguez (General Chair of IE'12), Davy Preuveneers (Short Papers Track co-Chair of IE'12) and Juan Carlos Augusto (Program co-Chair of IE'12) presenting a certificate to co-author Mathias Versichele.

The prize for the best demo was awarded to: "Personalized In-Vehicle Information Systems: Building and Application Infastructure for Smart Cars in Smart Spaces" by M.M. Moniri, Michael Feld, and Christian Müller. The photo in Fig. 3 shows, from left to right, Victor Zamudio Rodriguez (General Chair of IE'12), Davy Preuveneers (Short Papers Track coChair of IE'12) and Juan Carlos Augusto (Program coChair of IE'12) presenting a certificate to a colleague (Gerrit Kahl) in representation of the authors.

JAISE and IOS Press plan to extend this practice to other major events in related fields as a way to stimu- late high quality research around the world in the area of Ambient Intelligence and Smart Environments.

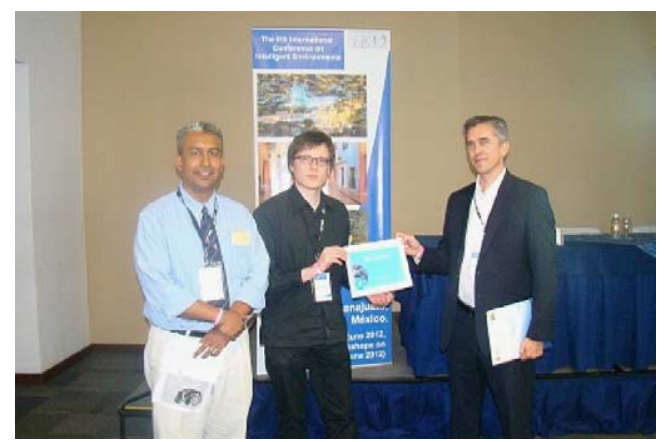

Fig. 1. Best Paper award.

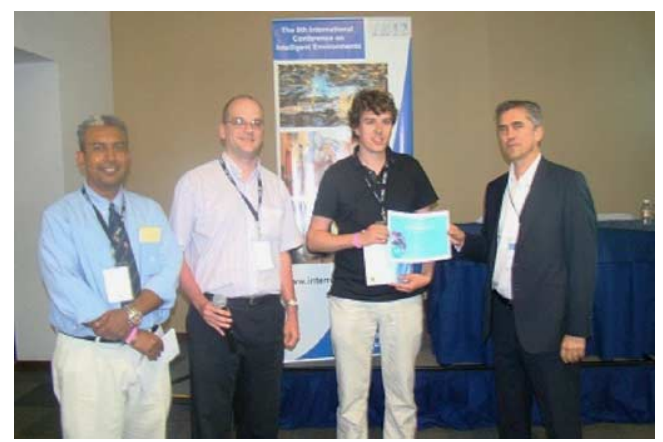

Fig. 2. Best Poster award.

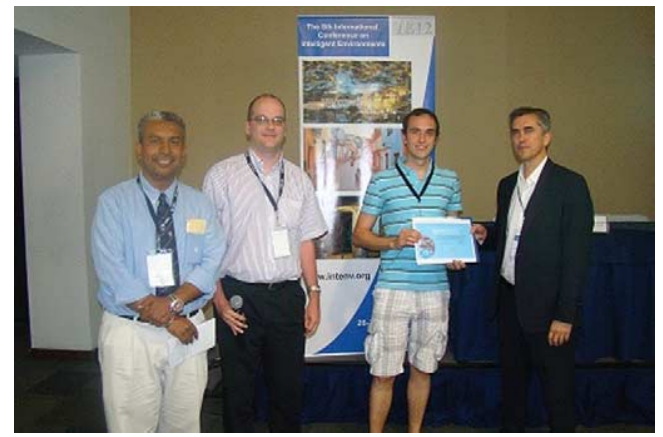

Fig. 3. Best Demo award. 